\title{
Article
}

\section{Effect of fullerene on the dispersibility of nanostructured lipid particles and encapsulation in sterically stabilized emulsions}

Kulkarni, Chandrashekhar Vishwanath, Moinuddin, Zeinab and Agarwal, Y

Available at http://clok.uclan.ac.uk/14971/

Kulkarni, Chandrashekhar Vishwanath ORCID: 0000-0002-5621-4791, Moinuddin, Zeinab and Agarwal, Y (2016) Effect of fullerene on the dispersibility of nanostructured lipid particles and encapsulation in sterically stabilized emulsions. Journal of Colloid and Interface Science, 480 . pp. 69-75. ISSN 0021-9797

It is advisable to refer to the publisher's version if you intend to cite from the work. http://dx.doi.org/10.1016/j.jcis.2016.07.008

For more information about UCLan's research in this area go to http://www.uclan.ac.uk/researchgroups/ and search for <name of research Group>.

For information about Research generally at UCLan please go to http://www.uclan.ac.uk/research/

All outputs in CLoK are protected by Intellectual Property Rights law, including Copyright law. Copyright, IPR and Moral Rights for the works on this site are retained by the individual authors and/or other copyright owners. Terms and conditions for use of this material are defined in the policies page. 


\title{
Effect of Fullerene on the Dispersibility of Nanostructured Lipid Particles and Encapsulation in Sterically Stabilized Emulsions
}

\author{
Chandrashekhar V. Kulkarni ${ }^{1 *}$, Zeinab Moinuddin ${ }^{1}$ and Yash Agarwal ${ }^{1,2}$ \\ ${ }^{1}$ Centre for Materials Science, School of Physical Sciences and Computing, University of Central \\ Lancashire, Preston PR1 2HE, United Kingdom. \\ ${ }^{2}$ Metallurgical Engineering \& Material Science Department, Indian Institute of Technology Bombay, \\ Powai-Mumbai, 400076, India. \\ *Corresponding author: cvkulkarni@uclan.ac.uk; Tel.: +44-1772-89-4339; Fax.: +44-1772-89-4981
}

\begin{abstract}
We report on the effect of fullerenes $\left(\mathrm{C}_{60}\right)$ on the stability of nanostructured lipid emulsions. These (oil-in-water) emulsions are essentially aqueous dispersions of lipid particles exhibiting self-assembled nanostructures at their cores. The majority of previous studies on fullerenes were focused on planar and spherical lipid bilayer systems including pure lipids and liposomes. In this work, fullerenes were interacted with a lipid that forms nanostructured dispersions of nonlamellar self-assemblies. A range of parameters including the composition of emulsions and sonication parameters were examined to determine the influence of fullerenes on in-situ and prestabilized lipid emulsions. We found that fullerenes mutually stabilize very low concentrations of lipid molecules, while other concentration emulsions struggle to stay stable or even to form at first instance; we provide hypotheses to support these observations. Interestingly though, we were able to encapsulate varying amounts of fullerenes in sterically stabilized emulsions. This step has a significant positive impact, as we could effectively control an inherent aggregation tendency of fullerenes in aqueous environments. These novel hybrid nanomaterials may open a range of avenues for biotechnological and biomedical applications exploiting properties of both lipid and fullerene nanostructures.
\end{abstract}

Keywords: nanostructured emulsions, lipid nanostructures, lipid self-assembly, fullerene, fullerenelipid interactions, fullerene aggregation, hybrid nanomaterials 


\section{Graphical Abstract:}

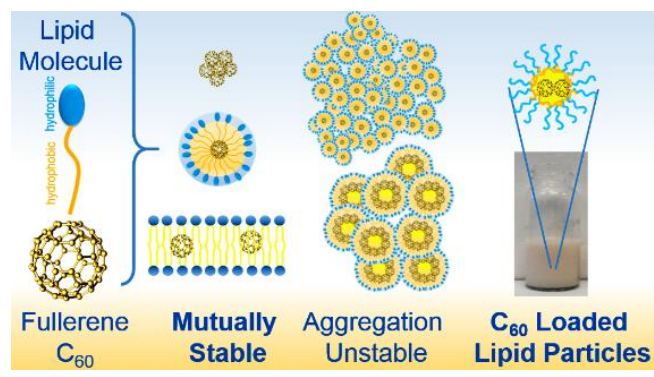

\section{Introduction}

Fullerenes, since their discovery $[1,2]$ have been considered as exciting materials for research and for the exploration of their novel applications [3-5]. However, they are sparingly soluble to almost insoluble in water (solubility $<10^{-9} \mathrm{mg} / \mathrm{L}$ ) [6] and toxic to cells [3]. These aspects turn into major bottlenecks for employing fullerenes in biotechnological applications. Some synthetic chemists, working in this direction, have been able to functionalize fullerenes and obtain their water-soluble derivatives $[4,7]$. However, in order to understand the biological activity of fullerenes and their potential therapeutic role, it is important to investigate their interactions with cellular membranes [8] than just solubility studies.

Considerable theoretical (simulation-based) [9-13] and experimental [8, 14] efforts have been taken to investigate fullerene interactions with biological as well as artificial membrane systems. These reports mainly involved studies with planar lipid bilayers, spherical micelles or vesicles. In this work we have taken a different approach and exploited dispersions of a lipid that forms non-lamellar selfassemblies. The main motivation behind this was twofold, 1) lyotropic liquid crystalline selfassemblies of non-lamellar (hexagonal and cubic) types, are highly attractive for biotechnological applications $[15,16]$, hence studying their interaction with fullerenes may outline the possibilities of developing novel hybrid nanomaterials similar to carbon nanotube-lipid hybrids we developed recently [17-19], and 2) due to their unique structure fullerenes could act as stabilizers for nanostructured lipid emulsions, by which an elegant properties of both - fullerenes and lipid nanostructures could be utilized for novel applications.

Non-lamellar self-assemblies employed in this work were prepared from a mixture of monoglyceride lipids. Due to their amphiphilic (amphi means two; i.e. both hydrophilic and hydrophobic) character, these lipids undergo spontaneous self-assembling in presence of aqueous medium [15]. The self- 
assemblies take forms of $0,1,2$, or 3 dimensional structures like, for example, inverse micelles, lamellar phase, hexagonal phase and cubic phases, respectively [15]. However, due to viscoelastic nature and variable domain consistency, some of these phases especially cubic ones, are hard to manipulate for certain applications. One of the best solutions researchers have found over this is to fragment these self-assemblies into a particle form using high energy input such as ultra-sonication [20-22]. This procedure is usually performed in presence of external stabilizers which shield fragmented particles and prevent their subsequent aggregation. Resulting dispersions are analogous to classical oil-in-water $(\mathrm{o} / \mathrm{w})$ emulsions but the oil phase, in this case, is replaced by a lipid selfassembly (Figure 1). Structural organization of lipid self-assemblies in the bulk (non-dispersed form) and emulsions (dispersed form) is usually determined using small angle X-ray scattering (SAXS) and transmission electron microscopy (TEM) techniques [23-25]. Depending on the type of internal selfassembly, the nanostructured emulsions are recognized as cubosomes for cubic nanostructures, hexosomes for hexagonal nanostructures and so on [15, 26-28]. The general term 'isasomes' meaning internally self-assembled particles (somes) is also often used in this context [26, 27, 29]. These emulsions exhibit a water-like fluidity desired for a range of high-throughput application including carrier systems for pharmaceutical and biomedical technologies [26, 27, 30, 31].

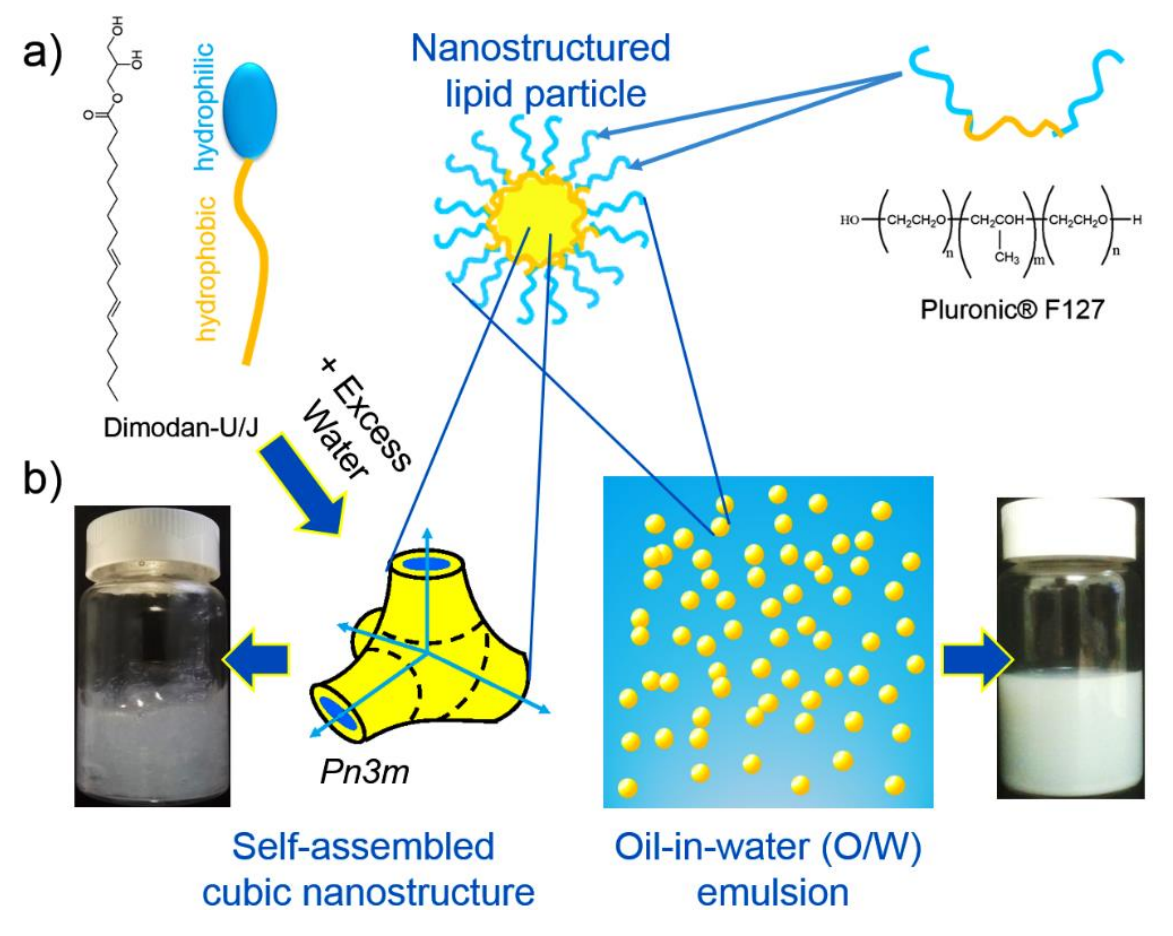

Figure 1. Composition of nanostructured emulsions: a) Chemical structures of Dimodan U/J (a lipid) and tri-block copolymer F127 (a stabilizer). Schematic diagrams shown near structures depict hydrophobic parts (yellow) and hydrophilic (blue) parts of molecules. b) In presence of water, lipid molecules self-assemble into cubic nanostructure ( $P n 3 m$ phase) which is fragmented into sub-micron sized particles that are stabilized using the F127 stabilizer [20]. Lipid cubic phase is usually highly 
viscous (shown on left) but the o/w emulsion has water-like fluidity (shown on right) improving their handling and applicability.

A wide range of stabilizers are employed for stabilizing aforementioned lipid nanoparticle emulsions [26]. Surfactant based stabilizers are more popular but solid particles have also been exploited with a varying level of success [26]. Emulsions stabilized by solid particles are termed generally as Pickering [32] or Ramsden-Pickering [33] emulsions. Clay platelets [34], food hydrocolloids [35, 36] and silica nanoparticles [37] are among such stabilizers, but in our recent work [18] we have employed carbon nanotubes (CNTs) for stabilizing nanostructured lipid particles. Pristine single-walled (SW) CNTs and functionalized multi-walled (MW) CNTs were employed successfully to stabilize internally selfassembled lipid particles [18]. More interestingly, concentrations of CNT-stabilizers were very low $(<10 \mu \mathrm{g} / \mathrm{ml})$. The lipid coating $[18,19]$ onto CNTs is believed to reduce their potential cytotoxicity given the fact that lipids are already among essential biomolecules in cells. The 'fullerene' is another carbon allotrope like CNTs, hence it is worth examining for stabilizing properties of nanostructured emulsions similar to CNTs [18]. Moreover, this study is also important in terms of understanding the interaction of fullerenes with a different (non-lamellar) type of model lipid membranes, which, to our knowledge has never been studied before.

\section{Materials and Methods}

\subsection{Materials}

Powdered buckminsterfullerene $\left(\mathrm{C}_{60}\right)$ and pluronic ${ }^{\circledR} \mathrm{F} 127\left(\mathrm{PEO}_{99}-\mathrm{PPO}_{67}-\mathrm{PEO}{ }_{99}\right)$ were obtained from Sigma-Aldrich Co. Ltd (Dorset, UK) and used without further treatment. Dimodan U/J® (DU) was generously provided by Danisco, Denmark; it is a commercial lipid containing distilled monoglycerides. Water was purified using Barnstead Nanopure, Thermoscientific (USA).

\subsection{Preparation of surfactant-stabilized (pre-stabilized) nanostructured lipid particles (Figure 1)}

Pre-stabilized lipid emulsions were prepared as follows: $500 \mathrm{mg}$ of molten lipid (DU) was dispensed into $20 \mathrm{ml}$ glass vial followed by addition of aqueous solution of $9.5 \mathrm{ml} 0.5 \mathrm{wt} \%$ F127 surfactant. Final emulsion had 5 wt.\% lipid; this concentration was used throughout the current work, unless mentioned specifically; also wt. (weight) $\%$ is represented with only $\%$ sign. The above mixture was ultra-sonicated (Sonics \& Materials Vibra-Cell VCX750, Jencons, UK) with $1 \mathrm{sec}$ pulses and $1 \mathrm{sec}$ delay times for (total) 20 min using 30\% (of the maximum) power. Final liquid crystalline phase 
within lipid particles is usually Pn3m cubic phase (Figure 1) and the particle size ranges in submicron to micron scales [26].

\subsection{Lipid emulsification using fullerenes}

A stock solution of fullerene $\left(\mathrm{C}_{60}\right)$ powder was prepared by dispersing $1.6 \mathrm{mg}$ of $\mathrm{C}_{60}$ in $50 \mathrm{ml}$ water. Probe ultra-sonication for $2 \mathrm{~min}$ at $35 \%$ (of the maximum) power facilitated the $\mathrm{C}_{60}$ dispersion in water. Fullerene dispersion stayed practically stable during the experimental time. This solution was subsequently diluted with water to obtain $1,5,10,20,25$ and $30 \mu \mathrm{g} / \mathrm{ml}$ concentrations of $\mathrm{C}_{60}$ in water. About $9.5 \mathrm{ml}$ of each of these dilutions were mixed with $500 \mathrm{mg}$ of molten DU and subjected to further sonication at higher power (40\%) for $2 \mathrm{~min}$ with an uninterrupted pulse (it should be noted that $40 \%$ power with continuous sonication worked better than $30 \%$ and $35 \%$ powers tested on similar dispersion systems). Resulting dispersions were analysed for the stability.

\section{Results and Discussion}

\subsection{Fullerenes as stabilizers?}

Upon absorption of water, DU forms cubic Pn3m nanostructure, which gets fragmented into submicron sized lipid particles due to sonication energy and consequential heating [20]. This results in a turbid (white) solution exhibiting water-like fluidity (Figure 1). If no stabilizer is added during the preparation, an emulsion gradually destabilizes due to aggregation of nanostructured lipid particles. Surfactant molecules, like F127 stabilize the particles' interfaces via steric stabilization [21] whereas solid particles like clay and silica stabilize via Pickering stabilization [32, 37, 38]. Some surfactant emulsions are co-stabilized by more than one type of mechanisms $[39,40]$. In our recent work we stabilized lipid based emulsions using a range of CNTs. The stabilization appears to occur via Pickering or a combination of the two mechanisms $[17,18]$. In case of fullerenes, we found that 25 and $30 \mu \mathrm{g} / \mathrm{ml}$ solutions of $\mathrm{C}_{60}$ stabilize the lipid particles into an o/w emulsion; however, lower $\mathrm{C}_{60}$ concentrations resulted in their aggregation. We have shown earlier that, the optimum ratio between lipid and stabilizer molecules is sometimes necessary for the final stability of emulsions [18]. Too little CNTs produced emulsions with lipid aggregates while too many CNTs contained aggregates with an excess of CNTs. Similar aggregates were also seen for fullerene concentrations lower than $20 \mu \mathrm{g} / \mathrm{ml}$ (Figure 2) dispersions. These aggregates assembled into rather big dark coloured lumps made of both fullerenes and lipids together. Spontaneous aggregation and clustering of fullerenes in aqueous media has been discussed by several researchers [7, 14, 41, 42]. Agglomeration and surface 
adhesion has also been demonstrated with surfactant micellar systems, cellular membranes as well as model lipid bilayers $[6,8,12,14]$.

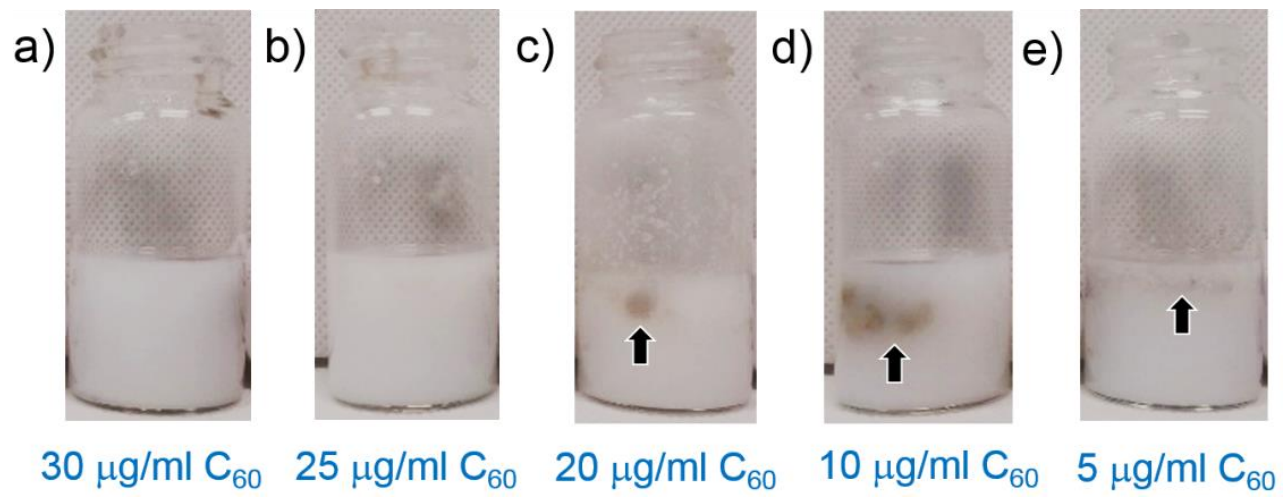

Figure 2. Stabilizing nanostructured lipid emulsions using fullerenes $\left(\mathbf{C}_{60}\right)$ : Mixtures of $5 \%$ lipid and $9.5 \mathrm{ml}$ distilled water were emulsified in presence various fullerene concentrations as follows: a) $30 \mu \mathrm{g} / \mathrm{ml}$, b) $25 \mu \mathrm{g} / \mathrm{ml}$, c) $20 \mu \mathrm{g} / \mathrm{ml}$, d) $10 \mu \mathrm{g} / \mathrm{ml}$ and e) $5 \mu \mathrm{g} / \mathrm{ml}$. Emulsions with a) $30 \mu \mathrm{g} / \mathrm{ml}$ and b) $25 \mu \mathrm{g} / \mathrm{ml} \mathrm{C} 60$ were produced successfully, however lower $\mathrm{C}_{60}$ concentrations struggled to form under similar experimental conditions. Unstable emulsions displayed dark coloured fullerene-lipid aggregates as visible in samples c), d), and e) (indicated by arrows).

We observed that the aggregation phenomenon expedites upon cooling of emulsions to room temperature while it is further accelerated by vigorous shaking, either manually or mechanically. In this context, the emulsions which were stable under hot conditions $\left(>60{ }^{\circ} \mathrm{C}\right)$, prepared from 25 and $30 \mu \mathrm{g} / \mathrm{ml}$ of $\mathrm{C}_{60}$ (Figure 2), also destabilized upon cooling and vigorous shaking. Initially formed small clusters perhaps act as nuclei or templates for subsequent aggregation into large masses [10]. The stability of lipid particle dispersions was further examined by modulating the concentration of fullerenes and ultra-sonication parameters (power and time); representative results are discussed below. High concentrations of $\mathrm{C}_{60}(3 \mathrm{mg} / \mathrm{ml})$ and ultra-sonication (1 sec on $1 \mathrm{sec}$ off) times of 5, 10 and $15 \mathrm{~min}$ at $35 \%$ power resulted in partially stable emulsions but they also contained small sized lipid+fullerene aggregates (see Supplementary material Figure SI01). The latter, upon gentle shaking agglomerated into a viscous lumps as noted earlier.

The viscous large aggregates (lumps) formed in each of the unstable/partially stable emulsions were harvested using a thin spatula on an absorbent paper and inspected for aesthetic properties. The size of lumps varied from $\sim 2 \mathrm{~mm}$ to $\sim 120 \mathrm{~mm}$ ( diameter) for a range of emulsions created using $\mathrm{C}_{60}$, whereas the colour also differed from emulsion to emulsion (Figure 3). The lump (for $30 \mu \mathrm{g} / \mathrm{ml}$ dispersion), allowed to stand overnight at room temperature displayed white dots. Apparently due to 
extreme hydrophobicity of fullerenes, the excess water ( $\mathrm{C}_{60}$ solubility $<10^{-9} \mathrm{mg} / \mathrm{L}[6]$ ) is expelled from the lump producing lamellar liquid crystals exhibiting minimal water content [43]. The remaining bulk part contains lipid cubic phase [43], which can be validated from characteristic high viscosity [43] and transparent colour due to its isotropic nature. Transparent cubic phase is also apparent from optical micrographs and visible inspection of lumps as shown in Figure 3. The presence of lamellar nanostructure in white dots was further verified using wide angle X-ray scattering (WAXS) technique (D2 Phaser, Brucker, UK) which displayed typical broad peak at 4.5 Å confirming fluid lamellar $\left(L_{\alpha}\right)$ nanostructure while other peaks (indicated by blue arrows in Figure 3d) elucidated crystalline lamellar $\left(L_{C}\right)$ polymorph. Upon heating above $60{ }^{\circ} \mathrm{C}$ for a few minutes (and cooling down to room temperature), the white dots got molten and the whole ensemble acquired almost uniform translucent colour (Figure 3e) depicting the existence of a cubic phase. However, during this process some spiky/spherulite structures were formed presumably due to the recrystallization - governed by combined association of fullerenes and lipids. The aggregates looked more dark (brown) with increasing fullerene concentrations (Figure 3e). This is attributable to the increased encapsulation of fullerenes. Observations of 'brown' instead of 'black' fullerene colour might be due to a molecular level complex formed of a fullerene and a lipid, which, however needs further confirmation using other characterization techniques and not discussed here anymore.

a)

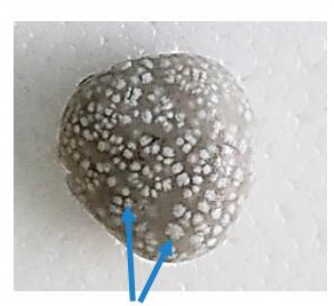

Lamellar nanostructure (white dots)

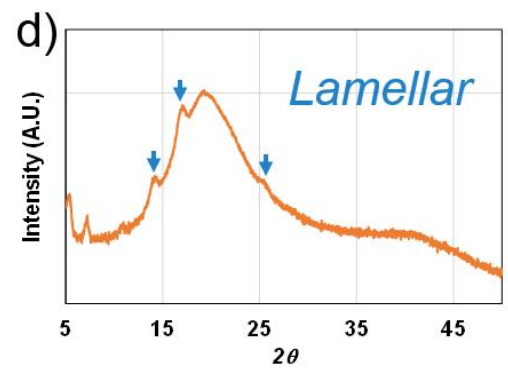

b)

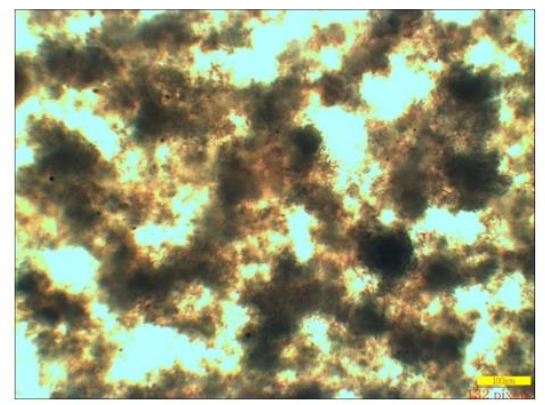

e)
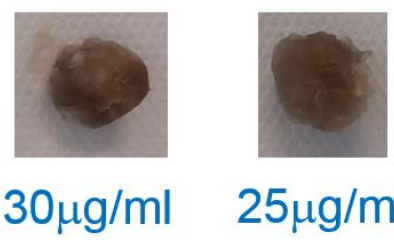

$25 \mu \mathrm{g} / \mathrm{ml}$
C)

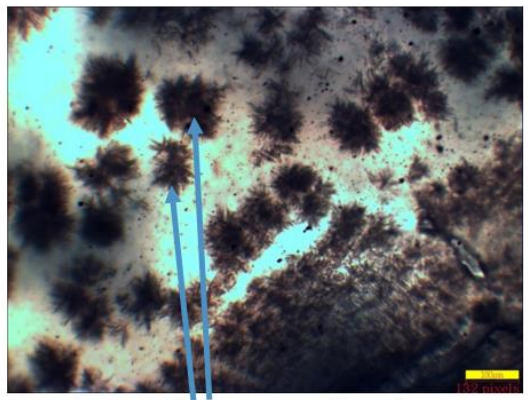

Lamellar nanostructure (spiky structures/spherulite)

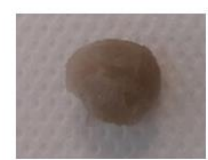

$20 \mu \mathrm{g} / \mathrm{ml}$

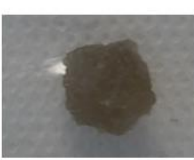

$10 \mu \mathrm{g} / \mathrm{ml}$

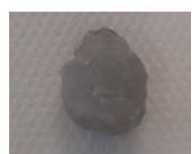

$5 \mu \mathrm{g} / \mathrm{ml}$

Figure 3. Analysis of $\mathbf{C}_{60}$-lipid aggregates: a) Lipid-fullerene aggregates harvested from an unstable emulsions depicted two different morphologies in the form of discrete white dots and dark coloured continuous phase. Optical micrographs b) and c) also validate this observation (scale bars indicate $100 \mu \mathrm{m}$ ) where apparent transparent phase consists isotropic cubic nanostructure while spiky structures/sphetulites represent lamellar nanostructure. d) WAXS studies support the existence of 
lamellar nanostructure: a broad peak of fluid lamellar phase $\left(L_{\alpha}\right)$ at $4.5 \AA$ and other peaks of crystalline lamellar nanostructure $\left(L_{C}\right)$ shown by arrows. e) Molten aggregates do not show white dots and appear darker (more brown) for higher fullerene concentrations demonstrating fullerene encapsulation therein.

\subsection{Mutual stabilization at very low lipid concentration}

Very low concentration of lipid $(0.1 \mathrm{wt} . \%)$ in pure water forms a milky dispersion, while (30 $\mu \mathrm{g} / \mathrm{ml})$ fullerene forms greyish brown solution subjecting to the sonication at $35 \%$ power (5 min at intermittent pulse) (Figure SI02). The mixtures of these show increasing brown colour when the concentration of fullerenes was increased (Figure SI02). Lumps or destabilization were not observed in these mixtures demonstrating that lipids and fullerenes act as mutual stabilizers to each other at such a low concentrations.

\subsection{Encapsulation of fullerenes in sterically stabilized emulsions}

The pluronic F127 is known for its efficient stabilization of lipid nanostructured particles [20, 26], hence we decided to investigate the combined effect of fullerenes and F127 in the current work. For this, $500 \mathrm{mg}$ lipid and $9.5 \mathrm{ml}$ of $30 \mu \mathrm{g} / \mathrm{ml}$ solution of $\mathrm{C}_{60}$ were mixed together followed by addition of F127 powder (to make total concentration of 0.5\% F127 in the final mixture). Ultra-sonication power of $35 \%$ for 10 min with intermittent pulses was adequate to form an aesthetically stable emulsion (Figure 4). Emulsions with other concentrations of fullerenes ( 3 and $300 \mu \mathrm{g} / \mathrm{ml}$ ) were also stable for long time (observed for about three weeks). High concentration of fullerenes imparted a brown tinge to the emulsion (Figure 4). A little sedimentation of fullerenes was observed for an emulsion made from $300 \mu \mathrm{g} / \mathrm{ml}$ concentration after about two days. The stability of these combined emulsions depicts that the stabilizing role of F127 dominates the destabilization character of fullerenes; in other words, the fullerenes have no detectable influence on the stability of nanostructured lipid particles. 

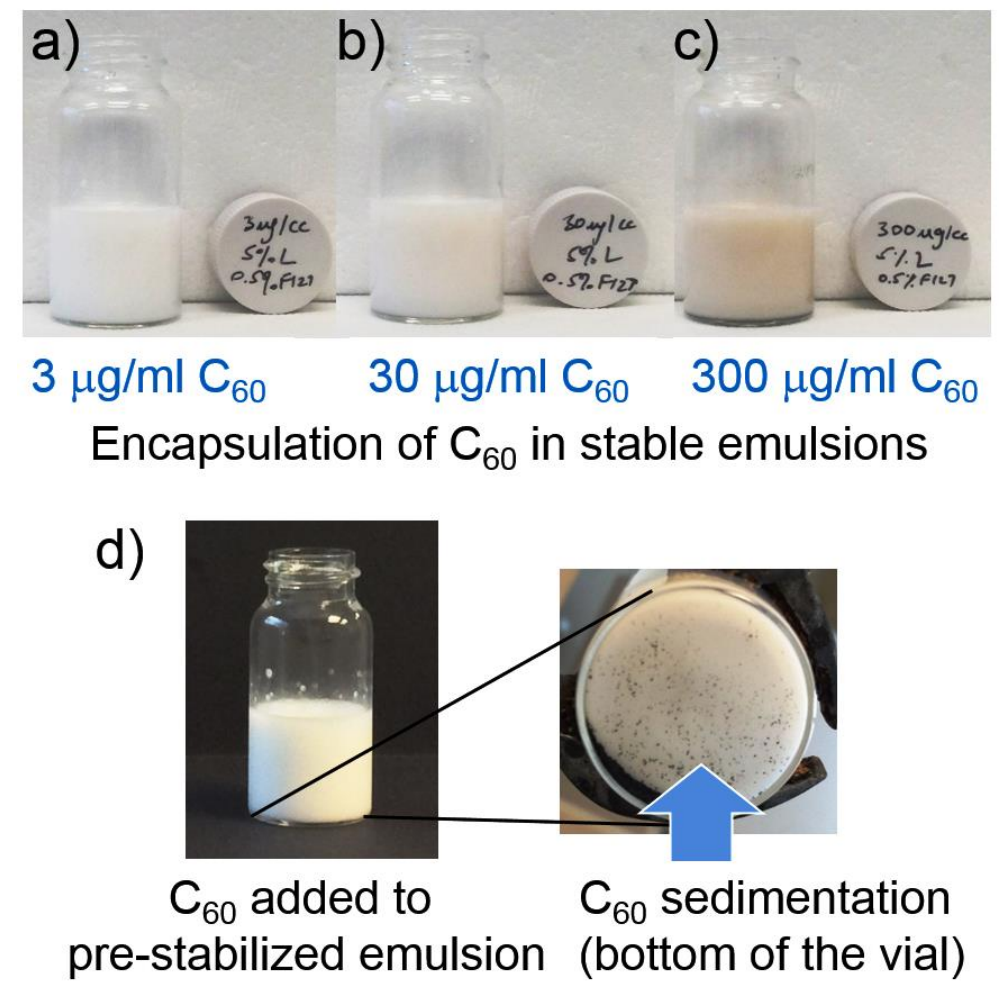

Figure 4. Emulsions with fullerene and F127 together: Emulsions prepared from $5 \%$ lipid, $0.5 \%$ F127 and varying concentrations of fullerenes a) $3 \mu \mathrm{g} / \mathrm{ml}$ b) $30 \mu \mathrm{g} / \mathrm{ml}$ and c) $300 \mu \mathrm{g} / \mathrm{ml}$ were stable for more than three weeks. High concentration of fullerene (in c) gave brown tinge to the emulsion. d) $\sim 1 \mathrm{mg}$ of fullerene powder mixed with pre-stabilized emulsion (using F127) did not show detectable influence on the emulsion characteristics; in contrast, remain separated as can be seen from the bottom of the sample vial.

F127 is a long tri-block copolymer (PEO99-PPO67-PEO99) that stabilizes lipid particles via steric stabilization [44] holding the nanostructured self-assembly in their cores (Figure 1). Intuitively, fullerenes encapsulate among these nanostructured cores during ultrasonication procedure. Clearly noticeable rise in dark colour, as a function of the concentration of fullerenes, in in-situ emulsions reveals increasing encapsulation of fullerenes (Figure 4). The possibility of fullerene adhesion at outer surfaces of lipid particles is practically low, simply because the fullerene-driven aggregation and destabilization of emulsions as seen for sole (no F127) fullerene-lipid dispersions (Figure 2 c, d, e) was absent here. Strong interaction forces of F127 molecules presumably seize the interface and form a steric cage around lipid particles averting fullerenes sticking to the particle surfaces. This was validated by a special experiment where a pre-stabilized emulsion (stabilized with F127 alone, see section 2.2 for more details) was topped with $1 \mathrm{mg}$ of fullerene powder and allowed to stand for 48 hrs at room temperature. Subsequently the mixture was shaken vigorously using a bench-top vortex unit. Despite of these treatments there was no noticeable change in milky-white emulsion colour as 
anticipated due to the fullerene entrapment within lipid particles (Figure 4d). Fullerenes were visibly sedimented at the bottom of the vial (Figure 4d). The experiment thus demonstrates that the fullerene encapsulation was only possible during in-situ ultrasonication.

\subsection{Potential pathways of fullerene-lipid stabilization and aggregation}

Fullerene- $\mathrm{C}_{60}$ is entirely made of carbon, where each of the carbon atoms binds to three other adjacent atoms via $\mathrm{SP}^{2}$ hybridization [45] making it extremely hydrophobic and insoluble in biologically relevant solvents [6]. Micellar systems formed by amphiphilic molecules like surfactants and lipids offer greater possibility of solubilizing them [6]. However, an inherent aggregation of fullerenes is almost unavoidable; nonetheless it can be limited to a certain sized clusters [6]. As revealed earlier, we were able to stabilize a dilute lipid solution (0.1 wt.\% lipid) using 5, 10 and $30 \mu \mathrm{g} / \mathrm{ml}$ fullerenes without forming visible fullerene (or fullerene-lipid) clusters (supplementary Figure SI02). This mutual stabilization is presumed to involve hybrid micelles (Figure 5a) formed by decoration of a) fullerene molecules and/or b) small aggregates of fullerenes by lipids [10]. The fullerene or aggregates are decorated, in principle, by hydrophobic lipid chains (facing fullerenes) while hydrophilic head groups face an aqueous environment [10]. Stabilization may also occur due to the spontaneous penetration of fullerenes in lipid bilayers, which ideally form a part of liposomes (vesicles) [8,46] (Figure 5a). Fullerene insertion in bilayers was previously investigated by several researchers $[6,8-10,12-14]$.

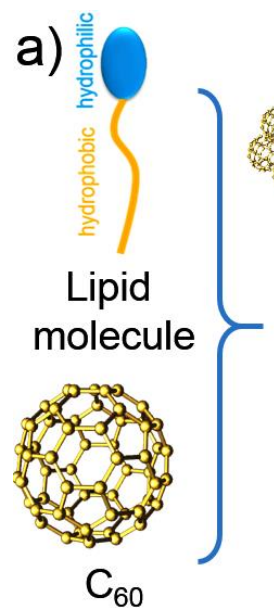

Fullerene

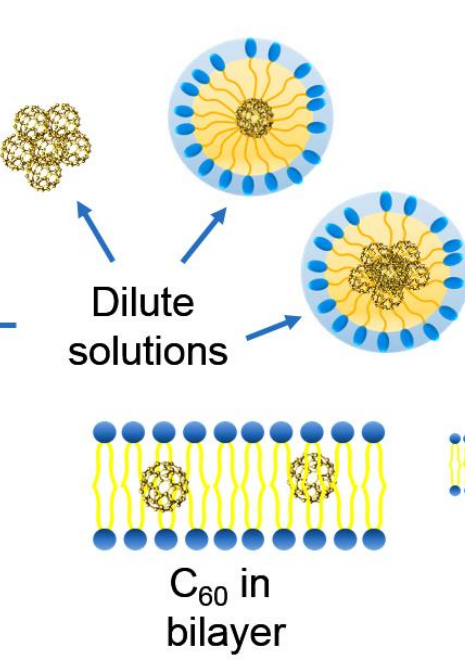

b)

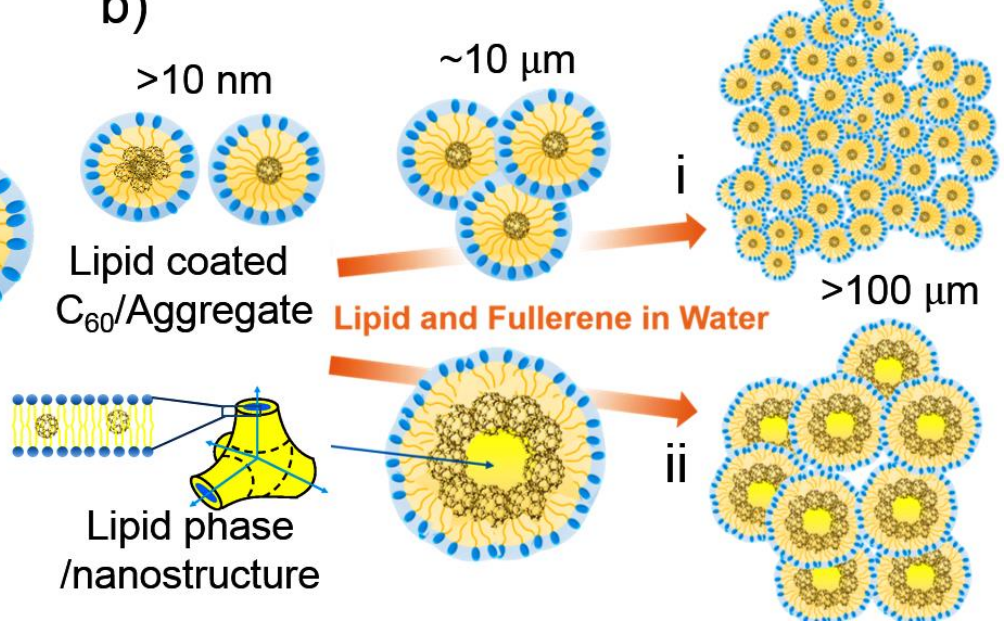

Figure 5. Pathways of emulsions a) stabilization and b) destabilization: a) Dilute lipid-fullerene dispersions are envisaged to be stabilized by lipid wrapping on fullerenes/small fullerene clusters and/or fullerene penetration in bilayers. b) Destabilization of (high lipid containing) emulsions is 
possibly driven by agglomeration of hierarchically structured particles comprised of lipid coated i) fullerene/fullerene aggregates and/or ii) fullerenes decorated fullerene-containing lipid nanostructure. Aforementioned low $(0.1 \%)$ lipid dispersions were stable, but 5\% lipid dispersions prepared using various fullerene concentrations faced severe destabilization which can be attributed partially to the following aspects: 1) self-aggregation of fullerenes in aqueous media [7, 42, 47], 2) adhesion of fullerenes to lipid membranes [8, 10, 46], 3) penetration of fullerenes [8] and/or small clusters [10] in membranes, 4) apparent high viscosity of liquid crystalline phase and 5) layer-by-layer growth of above aggregates during cooling and/or vigorous shaking where initial clusters act as templates (or nuclei) for the adhesion of additional layers [10]. However, these aspects need further confirmation with detail investigations. Based on our current observations we contemplate at least two pathways of destabilization - driven by an agglomeration of hierarchically structured particles, which, in turn are formed by amassing lipid coated i)fullerene/fullerene aggregates and/or ii) fullerenes decorated fullerene-containing lipid nanostructure (Figure 5b). These hypothess are supported by some representative evidences: Lipid wrapping on the fullerene surface was also discovered by Zhang et al. [10]; whereas Drasler et al. demonstrated the stabilization of MLV (multi-lamellar vesicle) bilayers by adhesion of fullerene clusters [8]. The agglomeration is apparently driven by strong hydrophobic forces among others, as depicted by the coexistence of (less hydrated) lamellar (Figure 3) together with (well-hydrated) cubic nanostructure; the latter is solely anticipated for pure lipid in water system at room temperature [43].

Despite of the above results, we have been successful in limiting the aggregation phenomenon by intercalating fullerenes in lipid particles during their steric stabilization. The steric stabilizer (F127) potentially blocks an aggregation of fullerenes on the surface of lipid particles. However, some of the fullerenes (and/or fullerene small clusters) categorically enter and stay within lipid particles whilst the self-assembled lipid nanostructure is fragmented using ultrasonication energy. Fullerene entrapment can be confirmed by noticeable increase in the darkness of emulsions with an increase in fullerene concentration (Figure 4). Further clustering of fullerenes, in this manner, almost vanished due to their encapsulation among lipid particles that remain kinetically stabilized by F127 molecules for rather long period. The difference in polydispersity determined by dynamic light scattering supports this observation (supplementary Figure SI03): mere $\mathrm{C}_{60}$-dispersions show wide range of particle size distribution while $\mathrm{C}_{60}-\mathrm{F} 127$ combined emulsions show rather narrow size distribution. 
The particle size in the $\mathrm{C}_{60}$-dispersion was as high as $10 \mu \mathrm{m}$ whereas particle size of $\mathrm{C}_{60}-\mathrm{F} 127$ combined emulsions stayed around and below $1 \mu \mathrm{m}$.

\section{Conclusions}

We investigated an effect of fullerene - $\mathrm{C}_{60}$ on dispersed non-lamellar lipid system, for the first time. We found that aqueous dispersions of low concentration of lipid $(0.1 \mathrm{wt} . \%)$ were mutually stabilized by fullerenes but higher lipid concentrations inclined towards destabilization. Aggregation of fullerenes in water $[42,47]$ is believed to be the primary cause of destabilization but the interaction with self-assembled lipid nanostructure and strong hydrophobic forces are presumed to promote it further [6, 10]. Large aggregates are believed to nucleate from small clusters [48] formed by lipid coating on fullerenes or fullerene self-assemblies triggering the destabilization. For this we propose at least two aggregation mechanisms as depicted in Figure 5b: i) lipid coated fullerene/fullerene aggregates and ii) fullerene decorated fullerene-containing lipid nanostructure - this ensemble may get enclosed by lipids, facing their head-groups towards water (Figure 5b). However, to validate proposed pathways these systems need to be investigated in greater details.

Although fullerenes themselves are not efficient stabilizers for high lipid emulsions, we were able to produce novel hybrid emulsions using an external stabilizer, albeit in presence of fullerenes. These sterically stabilized lipid particles were laden with various amounts (3, 30 and $300 \mu \mathrm{g} / \mathrm{ml}$ ) of fullerenes. As the fullerenes are encapsulated by lipid particles their apparent aggregation was highly suppressed. This can be primarily attributed to the overcoming of the hydrophobicity of fullerenes while preserving the system stability. With these in-situ emulsions we claim to have developed 'novel hybrid nanomaterials' where an elegant properties of both lipid particles as well as fullerenes are blended together in their application point of view. Moreover, the fullerene toxicity is anticipated to be reduced due to apparent coating with biocompatible lipid molecules (similar to our earlier work on coating of CNTs [18]).

To summarize, the current studies were focussed on three different lipid-fullerene systems: 1) dilute lipid solutions (0.1\% lipid) - mutually stabilized by fullerenes, 2) 5\% lipid dispersions - destabilized, owing to the fullerene-lipid aggregation and 3) fullerenes loaded sterically stabilized 5\% lipid emulsions - successful encapsulation of fullerenes by capping their aggregation and development of novel hybrid materials containing lipid nanostructures as well as fullerenes. Although detailed 
characterization and toxicity studies are required further, these novel nanomaterials are highly promising for a range of biotechnological and biomedical applications.

\section{Acknowledgements}

ZM would like to acknowledge UCLan's URIS-2015 Scheme for her financial support.

\section{References}

[1] H.P. Schultz, Topological Organic Chemistry. Polyhedranes and Prismanes, The Journal of Organic Chemistry 30(5) (1965) 1361-1364.

[2] H.W. Kroto, J.R. Heath, S.C. O'Brien, R.F. Curl, R.E. Smalley, C60: Buckminsterfullerene, Nature 318(6042) (1985) 162-163.

[3] B. Yadav, R. Kumar, Structure, properties and applications of fullerenes, International Journal of Nanotechnology \& Applications 2(1) (2008) 15-24.

[4] E. Nakamura, H. Isobe, Functionalized Fullerenes in Water. The First 10 Years of Their Chemistry, Biology, and Nanoscience, Accounts of Chemical Research 36(11) (2003) 807-815.

[5] H.S. Lee, S.C. Yoon, J. Lim, M. Lee, C. Lee, Novel 1,3-diketone modified fullerenes for organic photovoltaic cells, J Nanosci Nanotechnol 8(9) (2008) 4533-7.

[6] V.M. Torres, M. Posa, B. Srdjenovic, A.L. Simplicio, Solubilization of fullerene C60 in micellar solutions of different solubilizers, Colloids Surf B Biointerfaces 82(1) (2011) 46-53.

[7] I.A. Avilova, A.V. Chernyak, A.V. Zhilenkov, P.A. Troshin, V.I. Volkov, Self-organization of a water-soluble fullerene derivative studied by pulsed field gradient NMR spectroscopy, Mendeleev Communications 26(2) (2016) 146-148.

[8] B. Drasler, D. Drobne, A. Sadeghpour, M. Rappolt, Fullerene up-take alters bilayer structure and elasticity: A small angle X-ray study, Chemistry and Physics of Lipids 188(0) (2015) 46-53.

[9] J. Wong-Ekkabut, S. Baoukina, W. Triampo, I.M. Tang, D.P. Tieleman, L. Monticelli, Computer simulation study of fullerene translocation through lipid membranes, Nat Nano 3(6) (2008) 363-368. [10] S. Zhang, Y. Mu, J.Z.H. Zhang, W. Xu, Effect of Self-Assembly of Fullerene Nano-Particles on Lipid Membrane, PLoS ONE 8(10) (2013) e77436.

[11] J.H. Warner, M. Wilson, Elastic Distortions of Carbon Nanotubes Induced by Chiral Fullerene Chains, Acs Nano 4(7) (2010) 4011-4016.

[12] G. Rossi, J. Barnoud, L. Monticelli, Partitioning and solubility of C 60 fullerene in lipid membranes, Physica Scripta 87(5) (2013) 058503.

[13] R. Qiao, A.P. Roberts, A.S. Mount, S.J. Klaine, P.C. Ke, Translocation of C60 and its derivatives across a lipid bilayer, Nano Lett 7(3) (2007) 614-9.

[14] S.M. Santos, A.M. Dinis, F. Peixoto, L. Ferreira, A.S. Jurado, R.A. Videira, Interaction of fullerene nanoparticles with biomembranes: from the partition in lipid membranes to effects on mitochondrial bioenergetics, Toxicol Sci 138(1) (2014) 117-29.

[15] C.V. Kulkarni, Lipid crystallization: from self-assembly to hierarchical and biological ordering, Nanoscale 4(19) (2012) 5779-5791. 
[16] C.V. Kulkarni, W. Wachter, G.R. Iglesias, S. Engelskirchen, S. Ahualli, Monoolein: A Magic Lipid?, Phys Chem Chem Phys 13 (2011) 3004-3021.

[17] Y. Patil-Sen, A. Sadeghpour, M. Rappolt, C.V. Kulkarni, Facile Preparation of Internally Selfassembled Lipid Particles Stabilized by Carbon Nanotubes, Journal of Visualized Experiments (108) (2016) e53489.

[18] N.P. Gaunt, Y. Patil-Sen, M.J. Baker, C.V. Kulkarni, Carbon nanotubes for stabilization of nanostructured lipid particles, Nanoscale 7(3) (2015) 1090-1095.

[19] C. Paukner, K.K.K. Koziol, C.V. Kulkarni, Lipid nanoscaffolds in carbon nanotube arrays, Nanoscale 5(19) (2013) 8992-9000.

[20] S. Guillot, C. Moitzi, S. Salentinig, L. Sagalowicz, M.E. Leser, O. Glatter, Direct and indirect thermal transitions from hexosomes to emulsified micro-emulsions in oil-loaded monoglyceridebased particles, Colloids and Surfaces A: Physicochemical and Engineering Aspects 291(1-3) (2006) 78-84.

[21] J. Gustafsson, H. Ljusberg-Wahren, M. Almgren, K. Larsson, Cubic Lipid-Water Phase Dispersed into Submicron Particles, Langmuir 12(20) (1996) 4611-4613.

[22] J. Gustafsson, H. Ljusberg-Wahren, M. Almgren, K. Larsson, Submicron Particles of Reversed Lipid Phases in Water Stabilized by a Nonionic Amphiphilic Polymer, Langmuir 13(26) (1997) 69646971.

[23] A. Angelova, B. Angelov, R. Mutafchieva, S. Lesieur, Biocompatible Mesoporous and Soft Nanoarchitectures, Journal of Inorganic and Organometallic Polymers and Materials 25(2) (2015) 214-232.

[24] A. Angelova, B. Angelov, V.M. Garamus, P. Couvreur, S. Lesieur, Small-Angle X-ray Scattering Investigations of Biomolecular Confinement, Loading, and Release from LiquidCrystalline Nanochannel Assemblies, The Journal of Physical Chemistry Letters 3(3) (2012) 445457.

[25] B. Angelov, A. Angelova, V.M. Garamus, M. Drechsler, R. Willumeit, R. Mutafchieva, P. Štěpánek, S. Lesieur, Earliest Stage of the Tetrahedral Nanochannel Formation in Cubosome Particles from Unilamellar Nanovesicles, Langmuir 28(48) (2012) 16647-16655.

[26] C.V. Kulkarni, O. Glatter, Hierarchically Organized Systems Based on Liquid Crystalline Phases, in: N. Garti (Ed.), Self-Assembled Supramolecular Architectures: Lyotropic Liquid Crystals, John Wiley \& Sons, Inc.2012.

[27] C.V. Kulkarni, Hierarchically Structured Lipid Systems., in: G.C. Roberts (Ed.) Encyclopaedia of Biophysics, Springer Varlag., 2012.

[28] B. Angelov, A. Angelova, V.M. Garamus, S. Lesieur, Vehicles of inverted hexagonal liquid crystalline lipid phases self-assembled at room temperature, Journal of Optoelectronics and Advanced Materials 15(4) (2013) 211-215.

[29] A. Yaghmur, L. de Campo, L. Sagalowicz, M.E. Leser, O. Glatter, Emulsified Microemulsions and Oil-Containing Liquid Crystalline Phases, Langmuir 21(2) (2005) 569-577.

[30] A. Yaghmur, O. Glatter, Characterization and potential applications of nanostructured aqueous dispersions, Adv Colloid Interface Sci 147-148 (2009) 333-42.

[31] A. Angelova, B. Angelov, R. Mutafchieva, S. Lesieur, P. Couvreur, Self-Assembled Multicompartment Liquid Crystalline Lipid Carriers for Protein, Peptide, and Nucleic Acid Drug Delivery, Accounts of Chemical Research 44(2) (2011) 147-156.

[32] S.U. Pickering, Emulsions, J. Chem. Soc. 91 (1907) 2001. 
[33] W. Ramsden, Separation of Solids in the Surface-Layers of Solutions and 'Suspensions' (Observations on Surface-Membranes, Bubbles, Emulsions, and Mechanical Coagulation). Preliminary Account, Proceedings of the Royal Society of London 72(477-486) (1903) 156-164.

[34] S. Guillot, F. Bergaya, C. de Azevedo, F. Warmont, J.F. Tranchant, Internally structured pickering emulsions stabilized by clay mineral particles, J Colloid Interface Sci 333(2) (2009) 563-9. [35] E. Dickinson, Hydrocolloids as emulsifiers and emulsion stabilizers, Food Hydrocolloids 23(6) (2009) 1473-1482.

[36] N. Garti, D. Reichman, H.A.C.M. Hendrickx, E. Dickinson, L.K. Jackson, B. Bergenstahl, Hydrocolloids as Food Emulsifiers and Stabilizers, Food Struct 12(4) (1993) 411-426.

[37] A. Salonen, F.o. Muller, O. Glatter, Internally Self-Assembled Submicrometer Emulsions Stabilized by Spherical Nanocolloids: Finding the Free Nanoparticles in the Aqueous Continuous Phase, Langmuir 26(11) (2010) 7981-7987.

[38] N.P. Ashby, B.P. Binks, Pickering emulsions stabilised by Laponite clay particles, Physical Chemistry Chemical Physics 2(24) (2000) 5640-5646.

[39] K.L. Thompson, C.J. Mable, A. Cockram, N.J. Warren, V.J. Cunningham, E.R. Jones, R. Verber, S.P. Armes, Are block copolymer worms more effective Pickering emulsifiers than block copolymer spheres?, Soft Matter 10(43) (2014) 8615-8626.

[40] J.W. Salari, J. van Heck, B. Klumperman, Steric stabilization of Pickering emulsions for the efficient synthesis of polymeric microcapsules, Langmuir 26(18) (2010) 14929-36.

[41] A. Patnaik, Structure and dynamics in self-organized C60 fullerenes, J Nanosci Nanotechnol 7(4-5) (2007) 1111-50.

[42] Y.I. Prylutskyy, A.S. Buchelnikov, D.P. Voronin, V.V. Kostjukov, U. Ritter, J.A. Parkinson, M.P. Evstigneev, C60 fullerene aggregation in aqueous solution, Physical Chemistry Chemical Physics 15(23) (2013) 9351-9360.

[43] R. Mezzenga, C. Meyer, C. Servais, A.I. Romoscanu, L. Sagalowicz, R.C. Hayward, Shear Rheology of Lyotropic Liquid Crystals: A Case Study, Langmuir 21(8) (2005) 3322.

[44] A.J. Tilley, C.J. Drummond, B.J. Boyd, Disposition and association of the steric stabilizer Pluronic $®$ F127 in lyotropic liquid crystalline nanostructured particle dispersions, Journal of Colloid and Interface Science 392 (2013) 288-296.

[45] W. Kratschmer, L.D. Lamb, K. Fostiropoulos, D.R. Huffman, Solid C60: a new form of carbon, Nature 347(6291) (1990) 354-358.

[46] J. Zupanc, D. Drobne, B. Drasler, J. Valant, A. Iglic, V. Kralj-Iglic, D. Makovec, M. Rappolt, B. Sartori, K. Kogej, Experimental evidence for the interaction of C-60 fullerene with lipid vesicle membranes, Carbon 50(3) (2012) 1170-1178.

[47] D.P. Voronin, A.S. Buchelnikov, V.V. Kostjukov, S.V. Khrapatiy, D. Wyrzykowski, J. Piosik, Y.I. Prylutskyy, U. Ritter, M.P. Evstigneev, Evidence of entropically driven C60 fullerene aggregation in aqueous solution, The Journal of Chemical Physics 140(10) (2014) 104909.

[48] B. Angelov, A. Angelova, B. Papahadjopoulos-Sternberg, S.V. Hoffmann, V. Nicolas, S. Lesieur, Protein-Containing PEGylated Cubosomic Particles: Freeze-Fracture Electron Microscopy and Synchrotron Radiation Circular Dichroism Study, The Journal of Physical Chemistry B 116(26) (2012) 7676-7686. 